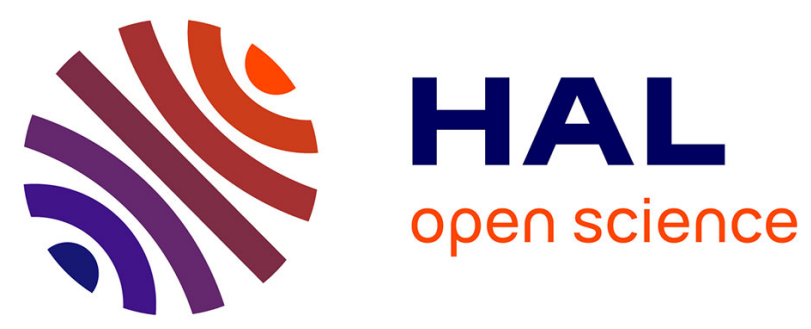

\title{
Simulating Turgor-Induced Stress Patterns in Multilayered Plant Tissues
}

Olivier Ali, Hadrien Oliveri, Jan Traas, Christophe Godin

\section{To cite this version:}

Olivier Ali, Hadrien Oliveri, Jan Traas, Christophe Godin. Simulating Turgor-Induced Stress Patterns in Multilayered Plant Tissues. Bulletin of Mathematical Biology, 2019, 81 (8), pp.1-23. 10.1007/s11538-019-00622-z . hal-02154814

\section{HAL Id: hal-02154814 https://hal.inria.fr/hal-02154814}

Submitted on 13 Jun 2019

HAL is a multi-disciplinary open access archive for the deposit and dissemination of scientific research documents, whether they are published or not. The documents may come from teaching and research institutions in France or abroad, or from public or private research centers.
L'archive ouverte pluridisciplinaire HAL, est destinée au dépôt et à la diffusion de documents scientifiques de niveau recherche, publiés ou non, émanant des établissements d'enseignement et de recherche français ou étrangers, des laboratoires publics ou privés. 


\title{
Simulating Turgor-Induced Stress Patterns in Multilayered Plant Tissues
}

\author{
Olivier Ali $^{1}$ (D) Hadrien Oliveri ${ }^{1} \cdot$ Jan $^{\text {Traas }^{1}} \cdot$ Christophe Godin $^{1}$
}

Received: 10 October 2018 / Accepted: 27 May 2019

(C) Society for Mathematical Biology 2019

\begin{abstract}
The intertwining between mechanics and developmental biology is extensively studied at the shoot apical meristem of land plants. Indeed, plants morphogenesis heavily relies on mechanics; tissue deformations are fueled by turgor-induced forces, and cell mechanosensitivity plays a major regulatory role in this dynamics. Since measurements of forces in growing meristems are still out of reach, our current knowledge relies mainly on theoretical and numerical models. So far, these modeling efforts have been mostly focusing on the epidermis, where aerial organs are initiated. In many models, the epidermis is assimilated to its outermost cell walls and described as a thin continuous shell under pressure, thereby neglecting the inner walls. There is, however, growing experimental evidence suggesting a significant mechanical role of these inner walls. The aim of this work is to investigate the influence of inner walls on the mechanical homeostasis of meristematic tissues. To this end, we simulated numerically the effect of turgor-induced loading, both in realistic flower buds and in more abstract structures. These simulations were performed using finite element meshes with subcellular resolution. Our analysis sheds light on the mechanics of growing plants by revealing the strong influence of inner walls on the epidermis mechanical stress pattern especially in negatively curved regions. Our simulations also display some strong and unsuspected features, such as a correlation between stress intensity and cell size, as well as differential response to loading between epidermal and inner cells. Finally, we monitored the time evolution of the mechanical stresses felt by each cell and its descendants during the early steps of flower morphogenesis.
\end{abstract}

Electronic supplementary material The online version of this article (https://doi.org/10.1007/s11538019-00622-z) contains supplementary material, which is available to authorized users.

$凶$ Olivier Ali

olivier.ali@inria.fr

1 Laboratoire de Reproduction et Développement des plantes, Univ Lyon, ENS de Lyon, UCB Lyon 1, CNRS, INRA, Inria, 69342 Lyon, France 
Keywords Computational biology $\cdot$ Biophysics

\section{Introduction}

Morphogenesis of multicellular organisms relies on two distinct and complementary cellular abilities: (i) growth - the ability of cells to expand and duplicate, and (ii) spatial awareness - the ability of cells to locate themselves in space. The latter is required to enable spatiotemporal regulation of the former. The strong reproducibility and robustness of developmental dynamics at the scale of organisms (Vogel 2013), despite stochasticity of cellular arrangements, suggest that spatial awareness relies on some kind of supracellular positional information field.

Such a concept has been first proposed and studied by pioneers such as Turing (1952) and Wolpert (1969). These explored a chemical spatial awareness theory; spatiotemporal regulation of growth is mediated by chemical substances called morphogens, see Green and Sharpe (2015) for a general introduction to these seminal works. Ever since, abundant experimental evidence for such biochemical mechanisms has been obtained.

In parallel, mechanical forces have been identified as cell fate regulators (Théry et al. 2006; Ladoux and Mège 2017; Vining and Mooney 2017) and it has been proposed that mechanical stress fields (the multidimensional generalization of forces) could also guide morphogenesis (Shraiman 2005; Irvine and Shraiman 2017; Keller 2012). In the context of plant tissues, this mechanical spatial awareness hypothesis is very appealing (Dumais 2007); epithelia are subject to pressure-induced stress field, presumably prescribed by tissue curvature. This echoes D'Arcy Thompson's dictum: '... the form of an object is a "diagram offorces", (Thompson 1917). Pressure-induced stress fields could provide mechanosensitive cells with instantaneous geometrical information about the shape of the tissue they are embedded in. Moreover, from a mathematical perspective, mechanical stresses are described as second-order symmetric tensors fields and therefore carry intrinsically more geometrical information (intensity, orientations and anisotropy) than scalar fields such as chemical concentrations.

Recently, stress sensitivity of plant cells has been studied in an increasing number of developmental systems: in the sunflower hypocotyl (Hejnowicz et al. 2000; Hejnowicz 2005) as well as in Arabidopsis thaliana meristem (Hamant et al. 2008; Uyttewaal et al. 2012) and hypocotyl (Sampathkumar et al. 2014), cell cortical cytoskeleton seems to polarize according to mechanical stress. In Arabidopsis, thwarting cytoskeleton dynamics (with depolymerizing drugs for instance) resulted in drastic growth-related effects: modification of cell wall deposition and cell shape (Corson et al. 2009), perturbed growth hormone signaling (Hamant et al. 2011), as well as transcriptional activation of cell wall modifying genes (Armezzani et al. 2018). Since the cytoskeleton is a central growth regulator, its stress sensitivity is a major entry point for mechanics into the control of morphogenesis. Cell division plane alignment in the Arabidopsis shoot apex has also been evidenced to rely upon mechanical stress patterns (Lintilhac and Vesecky 1984; Louveaux et al. 2016). Being able to measure mechanical stresses undergone by growing plant tissues would be a major step forward. Unfortunately, in vivo quantification of forces in plants is still an unresolved experimental challenge. 
To alleviate this limitation, modeling efforts have been initiated and simulating the mechanics of such systems has become an active field of research (Geitmann and Ortega 2009; Ali et al. 2014; Chickarmane et al. 2010).

Based on histological and mechanical properties of their constitutive cells, plant tissues can mechanically be compared to visco-elasto-plastic foams under pressure (Ortega 1985; Corson et al. 2009). Within these foams, since they are usually much thicker than the inner walls, the outer epidermal walls are supposed to bear most of the pressure-induced stresses, see Fig. 1b. Consequently, a common strategy in most of the existing modeling approaches is to restrict the influence of inner tissues to pressure forces applied onto the epidermis and to focus only on the mechanics of this outermost layer described as a pressure vessel. Such approaches either described the epidermis as a two-dimensional (2D) curved continuum (Hamant et al. 2008; Bozorg et al. 2014; Oliveri et al. 2018; Kierzkowski et al. 2012) or as a single layer of cells fixed on a flat surface (Sampathkumar et al. 2014; Sapala et al. 2018).

To go further, recent studies have attempted to grasp the complexity of fully $3 \mathrm{D}$ tissues through simulations based on either artificial structures (Mosca et al. 2017) or reconstructed ones from confocal images (Bassel et al. 2014; Mosca et al. 2017). However, it is not yet clear how the simple connection between tissue geometry and stress patterns, suggested by the pressure vessel model, is altered when inner walls are considered. The present work is an attempt to fill this gap. To this end, we tackle two questions: (i) to what extent the predicted stress patterns at the epidermal surface are modified when inner cells are considered as load-bearing? (ii) What are the characteristics (e.g., intensity, anisotropy, orientation) of the stresses borne by these inner cells?

To address these questions, we conducted numerical simulations of pressureinduced stress fields on 3D elastic structures at mechanical equilibrium. To this end, we used: (i) artificial structures generated from geometrical primitives such as spheres and cylinders, see Fig. 2b. (ii) Realistic ones obtained from 3D confocal stacks of real living plant tissues. More precisely, we generated meshes from segmented 3D images from a time series of the early steps of flower formation, see Fig. 2a. While studying the former enabled us to quantify the influence of inner load-bearing elements on known stress patterns, studying the latter yielded a (semi-)quantitative view of the stress patterns experienced by real tissues.

\section{Method and Model}

In this section, we detail our simulation pipeline. In the first subsection, we present our model and the method used to estimate mechanical equilibrium. In the second one, we describe how we generated triangular meshes from 3D images. In the last section, we detail the analysis procedure we applied to the simulations outputs. 


\subsection{Mechanical Modeling and Numerical Simulation of Pressurized Plant Tissues}

Static equilibrium of plant tissues under pressure Because growth-induced deformations in plant tissues are far slower than their elastic response to loading, plant cell growth is usually depicted as a quasistatic evolution (Dumais et al. 2006; Bozorg et al. 2016); the tissues are assumed at mechanical equilibrium at any moment. This mechanical equilibrium relies on the balance between pressure forces (due to the turgidity of cytosolic compartments) and the rheological response of the cell walls, characterized by the mechanical stress field at equilibrium and noted $\boldsymbol{\sigma}(\boldsymbol{x})$ hereafter. We assumed all cells to feature the same excess of pressure $P$ relative to the outside environment. Since these walls are thin $(\sim 0.5 \mu \mathrm{m})$ compared to the characteristic length of cells $(\sim 10 \mu \mathrm{m})$, we assumed plane stress conditions, i.e., we neglected the variations of the stress field across the walls and restricted its description to a tangential 2D second-order symmetric tensor field. Equations describing this equilibrium are gathered in system 1 (Landau and Lifshitz 1959):

$$
\begin{cases}\nabla \cdot \boldsymbol{\sigma}(\boldsymbol{x})=\mathbf{0} & \text { in } \Omega \\ \boldsymbol{\sigma}(\boldsymbol{x}) \cdot \hat{\boldsymbol{n}}(\boldsymbol{x})=P \hat{\boldsymbol{n}}(\boldsymbol{x}) & \text { on } \partial \Omega_{N} \\ \boldsymbol{u}(\boldsymbol{x})=\mathbf{0} & \text { on } \partial \Omega_{D}\end{cases}
$$

In system $1, \boldsymbol{u}$ stands for the displacement field and $\Omega$ for the continuum formed by the assembly of all the walls taken together. Domains $\partial \Omega_{N}$ and $\partial \Omega_{D}$ represent the boundaries of $\Omega$ where different conditions are enforced (subscripts $N$ and $D$, respectively, stand for Neumann and Dirichlet). Subdomain $\partial \Omega_{N}$ is the collection of walls where net pressure forces are acting, i.e., outermost walls of the tissue, while $\partial \Omega_{D}$ is the collection of walls assumed to be fixed, see Fig. 2e. Depending on the structure we investigated, the latter condition was not always enforced, i.e., $\partial \Omega_{D}=\emptyset$. When used, it was enforced by imposing a null displacement field $\boldsymbol{u}(\boldsymbol{x})$ on $\partial \Omega_{D}$, as prescribed by the third line of system 1 .

To complete the mechanical description, one needs to specify the constitutive relation between stress and strain within walls. For the sake of simplicity, we assumed this response to be linear elastic and isotropic. These assumptions yield the simplest rheological model for the cell walls: Hooke's law, depicted by Eq. 2, where $\mathbb{H}(\boldsymbol{x})$ stands for the cell wall elasticity tensor (Landau and Lifshitz 1959).

$$
\boldsymbol{\sigma}(\boldsymbol{x})=\mathbb{H}(\boldsymbol{x}): \boldsymbol{\varepsilon}(\boldsymbol{x})
$$

where ":" stands for the tensor double contraction. In the case of isotropic elasticity, the fourth-order elasticity tensor $\mathbb{H}$ is parameterized by only two variables: The Young's modulus $(Y)$ and the Poisson's ratio $(v)$. The spatial dependency of the elasticity tensor accounts for the fact that in some of our simulations, we allowed different values of Young's modulus for different cell walls.

Equations 1 and 2 constitute the core of our mechanical model. They are in accordance with other state-of-the-art modeling approaches within the field of plant tissue mechanics (Hamant et al. 2008; Bozorg et al. 2014; Bassel et al. 2014; Boudon et al. 2015; Mosca et al. 2017). Numerical values of the various parameters can be found, for 
each simulation, in the supplementary materials. Across our various simulations, we tested pressure values from 0.1 to $10 \mathrm{MPa}$ and Young's moduli between 1 and $45 \mathrm{MPa}$. We took care to use values compatible with experimental measures (Chanliaud et al. 2002; Beauzamy et al. 2015; Milani et al. 2011; Kierzkowski et al. 2012) and existing models (Hamant et al. 2008; Bozorg et al. 2014; Sampathkumar et al. 2014; Hervieux et al. 2016; Mosca et al. 2017) for realistic structures as well as for abstract ones, see section $\mathrm{C}$ of the supplementary materials for details.

Numerical estimation of turgor-induced stress fields The numerical framework we used to conduct our simulations relies on two main open-source softwares: OpenAlea (Pradal et al. 2008) and Sofa (Faure et al. 2012). The former is used to describe the tissues and to generate the data structure, the latter to handle numerical simulations. The general workflow of our framework is detailed in Boudon et al. (2015).

We chose to discretize the continuum composed by all the walls $(\Omega$ in system 1 ) using first-order finite elements (FEs). Because bending stiffness can be neglected for turgid cells (Weber et al. 2015; Bozorg et al. 2014), we used membrane elements. Mechanical equilibrium is computed by solving the weak form of system 1, which is achieved through gradient descent. For faster convergence and stability, we used a damped implicit scheme available in the Sofa Module Compliance and based on the methods described in Tournier et al. (2015).

\subsection{Realistic Meshes Production}

Mesh production from segmented 3D images In order to generate accurate simulations of realistic tissues, we needed $F E$ meshes that satisfied two requirements: on one hand, for the sake of stability and convergence, they had to be composed of elements as regular as possible. In the present case, our triangular elements have to be as close as possible to equilateral triangles. On the other hand, they had to account for tissues cellularization and layering, which imposed topological constrains on the tiling. Production of meshes managing these constraints was achieved through the use of the DRACO-STEM library, described in Cerutti et al. (2017). This library provides tools to generate regular cell-preserving triangular meshes from 3D segmented images where cells are represented as sets of voxels labeled with the same identifier (Id). Such segmented images can be produced from 3D confocal stacks of real tissues as well as from artificially generated images.

3D segmented images from confocal microscopy Segmented images from the early steps of a time series of a flower bud formation were obtained through the Mars-Alt pipeline (Fernandez et al. 2010) and curated manually. Applying this segmentation pipeline to consecutive steps of a time series enabled us to also compute the lineage of cells within the structure. Based on this lineage information, we were able to track the developmental history of characteristic zones defined on the last time step, see Fig. $2 \mathrm{a}$. 
Note that because the quality of the segmenting procedure depends heavily on the intensity signal within the initial 3D images, we were only able to reconstruct the two outermost cell layers of each time step of the flower bud time series, see Fig. 2c, d.

3D segmented images from geometrical primitives To complete this set of realistic meshes, we also developed algorithms to generate 3D segmented images of artificial geometrical volumes decomposed into cells. The generation of such cellularized geometrical structures involves two steps: (i) first surface meshes encompassing the desired volumes are generated from a 3D computer graphics software (in our case Blender). (ii) Then, the volumes defined by the meshes are populated with points, serving as seeds for a 3D centroidal Voronoi tessellation algorithm (see supplementary information).

For the present study, we generated meshes from the concatenation of simple primitives such as spheres and cylinders. We limited our analysis to three types of artificial structures: (i) a sphere, (ii) a "pill", i.e., a cylinder closed by two hemispheres, (iii) and a "bowling pin" composed by the concatenation of two spheres of different radius, see Fig. 2b. Each structure was decomposed into five hundred cells generated from points disposed homogeneously and randomly within the considered volumes.

Structures generated from artificial geometrical volumes present some advantages compared to realistic ones. First, contrary to what happens with confocal images, there is no depth limitation with artificially generated images. From such images, we were therefore able to generate 3D structures completely filled with cells and not limited to the two outermost layers, see Fig. 2c, d. Secondly, since we restricted our analysis to axisymmetric, closed, structures; pressure forces being well balanced in such structures we did not have to fix the boundaries $\left(\partial \Omega_{D}=\emptyset\right.$ in (1)) in our simulations.

Different versions of each structure to analyze the stress field To probe the influence of inner walls on the mechanical stress pattern borne by the outermost surface, we generated for each structure several versions by varying the number of inner cell layers.

For the realistic structures, containing two cell layers, we generated three variants, see Fig. 2c, d: the first one, noted Shell, consists of the outermost cell walls of the epidermis, called $L_{1}$ outermost periclinal walls. In the second one, noted $L_{1}$-only, all the walls surrounding the epidermal cells were taken into account, namely the $L_{1}$ outermost periclinal, the $L_{1}$ anticlinal and the $L_{1}-L_{2}$ periclinal walls. Finally, in the most detailed version, noted $L_{1}-L_{2}$, all the walls from the two outermost cell layers were considered. This includes, on top of the previously cited, the $L_{2}$-anticlinal and the $L_{2}$ periclinal walls.

With abstract structures, we were not limited to the two outermost layers and therefore added a fourth version: the Full one, encompassing all the walls from the two outermost layers and including also all the remaining inner walls.

\subsection{Analysis of Simulation Outputs}

Normalized stress field To probe the influence of inner tissues on stress patterns, we compared stress patterns between different versions of the same structure with more or 
less inner walls. We were therefore more interested in the comparison between stress patterns rather than by their absolute values. We, therefore, normalized every stress field by the pressure value used to generate them:

$$
\tilde{\sigma}=\frac{\sigma}{P}
$$

This yields a dimensionless tensorial field, hereafter referred to as the normalized stress field, which depends mostly on the geometry, the topology and the rigidity of the underlying network of cell walls. Note that for the sake of simplicity, we will drop the tilde notation in the rest of the text, since all stress quantities we will discuss will be normalized.

Stress field quantifiers In the plane stress approximation, mechanical stresses within cell walls can be expressed as 2D second-order symmetric tensors. As such, mechanical stresses are fully parameterized by a set of three independent variables, we call quantifiers: their intensity, anisotropy and orientation. Such tensors can also be uniquely decomposed into isotropic (iso (.)) and deviatoric $(\operatorname{dev}(\cdot))$ parts: $\sigma=$ iso $(\sigma)+\operatorname{dev}(\sigma)$.

It appeared fundamental to look at intrinsic physical quantities, which do not rely on an arbitrary coordinate system and are directly available to cells. We therefore focus our attention on intensity and anisotropy (respectively noted $\sigma$ and $\alpha_{\sigma}$ hereafter). These two scalar properties can be extracted from the isotropic/deviatoric decomposition previously mentioned. We chose to use the following definitions for these two quantifiers:

with the 2D second-order matrix norm defined as the Frobenius norm divided by $\sqrt{2}$ : $\|\boldsymbol{T}\|=\sqrt{\operatorname{tr}\left(\boldsymbol{T}^{2}\right) / 2}$. The variables $\sigma_{\mathrm{M}}$ and $\sigma_{\mathrm{m}}$, respectively, stand for the maximal and minimal eigenvalues of the normalized stress tensor $\sigma$ considered $\left(\sigma_{M} \geq \sigma_{\mathrm{m}}\right)$.

The choice of the specific expressions in (4) was made such that our quantifiers meet the usual definitions of intensity (mean value of the eigenvalues: $T_{\mathbf{M}}+T_{\mathbf{m}} / 2$ ) and anisotropy (ratio between the eigenvalues difference and sum: $T_{\mathbf{M}}-T_{\mathbf{m}} / T_{\mathbf{M}}+T_{\mathbf{m}}$ ) in the case of tensile stresses. To avoid confusion, we checked that no significant compressions could be observed in our simulations (see subsection C.6 in the supplementary materials for details). Moreover, the use of absolute values enables us to define the intensity as the natural extension of the usual vector norm to second-order symmetric tensors.

With these definitions, the normalized stress field can be expressed, in the most general way, as follows:

$$
\boldsymbol{\sigma}= \pm \sigma\left(\boldsymbol{I}_{d}+\alpha_{\sigma}\left(\boldsymbol{P}_{\theta_{\sigma}}-\boldsymbol{P}_{\theta_{\sigma}+\pi / 2}\right)\right)
$$


The angle $\theta_{\sigma}$ quantifies the direction associated with the largest eigenvalue of $\boldsymbol{\sigma}$ and $\boldsymbol{P}_{\theta}=\hat{\boldsymbol{e}}_{\theta} \otimes \hat{\boldsymbol{e}}_{\theta}$ is the projector on the direction given by the unit vector $\hat{\boldsymbol{e}}_{\theta}=[\cos (\theta) \sin (\theta)]^{t}$. The sign $( \pm)$ in front of the rhs of 5 corresponds to the sign of $\operatorname{tr}(\sigma)$.

Cell-averaged stress field We used subcellular resolution meshes for our simulations, where each wall was decomposed into triangles (around 20 on average, see tables 1 and 2 in the supplementary materials). The stress tensors were computed within each of these triangles. In order to smooth out numerical fluctuations due to mesh topology irregularities between neighboring triangles, we applied a closest-neighbors averaging filter on these tensors (see supplementary materials, section B) as a preprocessing step (i.e., prior to any other analysis) on all of our raw data generated by the simulation pipeline.

Since we focused the present analysis on tissue stress patterns, we ignored stress variations at the subcellular level in most of our analyses. Therefore, we averaged our normalized stress field quantifiers $\left(\sigma, \alpha_{\sigma}, \hat{\boldsymbol{e}}_{\theta_{\sigma}}\right)$ over each cell wall type for each cell (the cell wall-averaged versions of the quantifiers are denoted with a bar: $\bar{\sigma}, \bar{\alpha}_{\sigma}, \hat{\boldsymbol{e}}_{\bar{\theta}_{\sigma}}$ ); e.g., epidermal cells in the $L_{1}$-only, $L_{1}-L_{2}$ and full versions of a geometrical structure feature three sets of normalized stress invariants one for the outermost periclinal walls, one for the $L_{1}-L_{2}$ periclinal walls and one for its anticlinal walls.

\section{Results}

We focused on three aspects in our analysis: (i) the influence of inner cell walls on the outermost periclinal stress pattern. (ii) The characteristics of the stresses borne by inner walls and (iii) finally, the spatiotemporal sorting/clustering of cells based on the characteristics of the mechanical stress they experience.

\subsection{Influence of the Inner Cell Walls on the Outermost Periclinal Stress Field}

Taking inner walls into consideration diminishes stress field heterogeneity and anisotropy in the outermost walls To estimate the mechanical influence of inner walls, we compared the stress patterns borne by $L_{1}$ outermost periclinal walls between various versions (shell, $L_{1}$-only, $L_{1}-L_{2}$ and full) of the same structure (sphere, pill and bowling pin). For each version of each structure, we monitored the spatial variations of the stress field. To do so, we looked at relative spatial fluctuations of stress intensity, quantified by its cell-to-cell standard deviation divided by its mean value $(\Delta \sigma / \bar{\sigma})$. As Fig. 3a shows, these fluctuations are decreasing as we are considering an increasing number of inner walls. We also monitored the anisotropy of the $L_{1}$ outermost periclinal stress field and observed that it was also a decreasing function of the number of considered inner walls, see Fig. 3b. The sphere structure, represented by blue graphs in Fig. 3a, b, serves as a reference; stresses on an exact sphere should remain homogeneous and isotropic for any version, the slight fluctuations and anisotropy shown on the blue graphs of Fig. 3a, b are due to the discrepancy between discrete meshes and 
the exact sphere. High variations observed on the two other structures (red and green graphs) appear therefore significant.

Put together, these two observations suggest that the mechanical influence of inner walls tends to homogenize the stress pattern borne by outermost periclinal walls and make it more isotropic.

\section{Rigidity differential between inner and outer walls increases stress field hetero-} geneity and anisotropy in outermost walls One key assumption of the pressurized shell model is that inner walls can be disregarded as mechanical load-bearer since they are softer than outermost ones. To test this hypothesis, we conducted simulations on the full versions of our geometrical structures for various values of the rigidity ratio between the outermost periclinal walls and the inner ones: $r_{R}=Y_{\text {out }} / Y_{\text {in }}$, where $Y_{\text {out }}$ and $Y_{\mathrm{in}}$, respectively, stand for the Young's moduli of the $L_{1}$ outermost periclinal walls and the one of all the other walls. For each value of this ratio, we computed the mean intensity value of the normalized stresses borne by each type of cell within the full version of a given structure and compared it to the mean intensity value of the stress field developed within the shell version of the same structure: $\bar{\sigma}_{\text {full }}(\mathrm{cw}$ type $) / \bar{\sigma}_{\text {shell }}$ (see Fig. 3c for a visual representation). As the rigidity ratio between outermost and inner walls increases, the intensity ratio between stress borne by outermost and inner walls grows accordingly. Conversely, when all cell walls feature the same rigidity value, stress intensity is uniformly distributed among them. As expected for springs arranged in parallel and loaded with a common force, Fig. 3c suggests that relative distribution of stress between outermost and inner load-bearing elements corresponds to the relative distribution (ratio) of rigidities.

In meristematic tissues, outer walls are thicker than inner ones (see supporting material of Kierzkowski et al. 2012). Structural stiffness of a plane is proportional to its thickness (Landau and Lifshitz 1959). Therefore, if composition does not differ significantly from one wall to another, one can assume that thickness ratio translates directly into stiffness ratio between outer and inner walls which should roughly lies between 3 and 10 (Kierzkowski et al. 2012 and unpublished observation made in our laboratory). Figure $3 \mathrm{c}$ shows that the corresponding ratios between outermost over inner stress intensities lies within the same range and that outermost stress intensity represents $60 \%$ of what it would be in the shell version.

Finally, increasing standard deviation with rigidity ratio, accounted for by increasing error bars in Fig. 3c, suggests that rigidity differential not only increases heterogeneity between outermost and inner stress fields, but also increase heterogeneity within the outermost periclinal stress field itself. This is confirmed by Fig. 3d, e where outermost periclinal stress intensity fluctuations and anisotropy are, respectively, plotted as function of the rigidity differential.

Considering inner walls drastically reduce outermost stress intensity in negatively curved regions When surfaces grow into complex shapes, folding and crease formation are of prime importance because they define frontiers between organs. One output of the pressurized shell model is that creases, which correspond to zones of negative Gaussian curvature, feature high-intensity and highly anisotropic stresses (Bozorg et al. 2014). 
When applied to a realistic structure displaying such negatively curved regions, our simulation pipeline produced a counterintuitive result; while creases indeed supported high-intensity and highly anisotropic stresses on the shell version of the structure (Fig. 4a), they supported low-intensity (but still highly anisotropic) stresses in the $L_{1}$ only (Fig. 4b) and $L_{1}-L_{2}$ (Fig. 4c) versions. As seen in Fig. 4e, while stress relative intensity in the frontier zone is, on average, twice as big as in the central zone within the shell version; it decreases to almost half of the central zone mean value in the $L_{1}$-only version and to roughly $75 \%$ of it in the $L_{1}-L_{2}$ version.

Despite this strong influence of inner walls on the relative values of stress intensity between zones, Fig. $4 \mathrm{f}$ shows that anisotropy remains maximal in the frontier zone, no matter if the inner walls are considered or not. Similarly, stress orientation patterns did not change drastically between the various versions, see supplementary Fig. 1.

\section{Stress intensity in outermost walls correlates with the area of periclinal cell faces} Another feature of the $\mathrm{L}_{1}$ outermost periclinal stress pattern revealed by our simulations on realistic structures concerns the correlation between the stress intensity borne by outermost periclinal walls and their surface area, see Fig. 5a, b: in the surface area vs. stress intensity space, points representing cells from the epidermis are distributed along some saturation curve. In other words, our simulations show that bigger epidermal cells experience higher stress intensities in their outermost periclinal walls. Although this correlation seems also present in the $L_{2}$ layer, the saturating behavior is far less obvious, see the yellow point cloud in Fig. 5a. Stress intensity and surface area also appeared correlated in the $L_{2}$ layer, but with a less obvious saturating behavior. This "stress intensity-surface area" correlation vanishes obviously in the Shell versions of our structures, see Fig. 5c, demonstrating that this feature emerges from the consideration of inner walls.

Boundary conditions imposed on the innermost walls impact stress patterns at the surface The choice of fixed boundary conditions was imposed by our experimental data. As mentioned above, when applied to 3D fluorescence intensity images, our segmentation algorithms could only reconstruct faithfully the two outermost cell layers. As Fig. 7a, b shows, the resulting structures correspond to asymmetric concave hulls. In vivo, these layers are anchored to inner tissues. To take into account the influence of these inner tissues, we fixed the innermost points of the outer two layers in our simulations. This was done using Dirichlet boundary conditions, see third line of eq.1.

To assess the influence of such boundary conditions on the outermost periclinal stress pattern, we compared stress quantifiers (i.e., relative intensity $\bar{\sigma}$ and anisotropy $\bar{\alpha}_{\sigma}$, averaged over the outer periclinal wall of each epithelial cell) between various versions of the same 3D structure ( $L_{1}$-only, $L_{1}-L_{2}$, full), where the innermost points have been fixed, with the same quantifiers $\left(\bar{\sigma}^{\star} \bar{\alpha}_{\sigma}^{\star}\right)$ computed on the full version of the same structure without fixed innermost walls, see Fig. 6.

Figure 6 shows that fixed boundary conditions have a strong influence on the outermost stress pattern. As we considered more inner layers, this influence was reduced. Note that Fig. 6c, d show that depending on their curvature, various regions-of the bowling pin structure-are not impacted similarly by boundary conditions. 


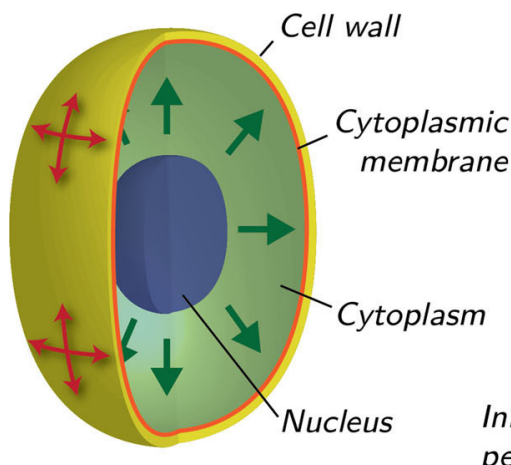

(a)

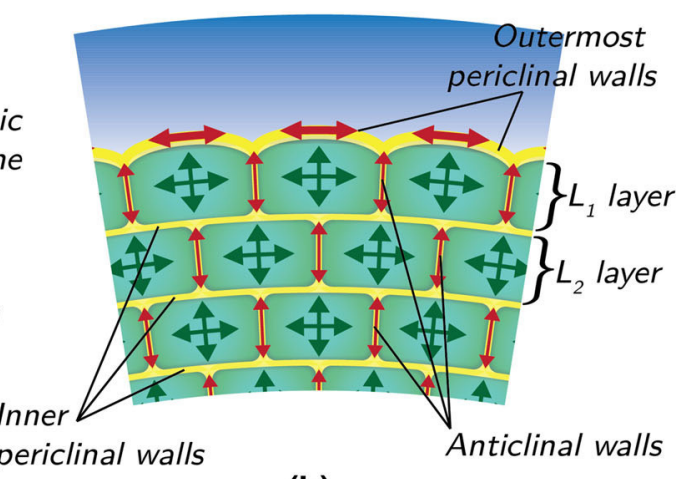

(b)

Fig. 1 Schematic description of plant cells and tissues. a Sketch of a plant cell. b Plant cells glued together by their cell walls, forming a tissue. In both $\mathbf{a} \mathbf{b}$, the green and red arrows, respectively, depict turgor pressure forces and response stresses within the cell walls

\subsection{Characteristics of the Inner Stress Field}

Anticlinal stresses are mostly oriented in the apicobasal direction Our simulations show that stresses borne by anticlinal walls are, when averaged within each cell, mainly aligned in the apicobasal direction (see Fig. 7a-d) and feature higher anisotropy compared to outer periclinal walls, see Fig. 7c. This suggests that stresses borne by anticlinal walls directly balance pressure forces normal to the outermost periclinal surface.

Negatively curved regions experience low-intensity anticlinal stresses The equilibrium of pressure forces and elastic stress highly depends on the curvature of the considered surface. As reported in the previous subsection, when inner walls were considered in our simulations, epidermal cells in negatively curved regions displayed low-intensity stresses on their outermost periclinal faces. Interestingly, the same cells also featured low-intensity stresses on their anticlinal faces, see Fig. 7e, f. Frontier zones between organs and the meristem are, therefore, mechanically characterized by low-intensity global stresses.

\subsection{Morphologically Distinct Regions Within a Tissue Experience Specific Stress Patterns}

Mechanical stress characteristics vary significantly between morphologically different regions For each cell of our 3D realistic structures, we computed a set of stress characteristics: The relative intensity and anisotropy averaged over their outermost periclinal wall and the relative stress intensity averaged over their anticlinal walls. From the previous sections, we expected these characteristics to vary from one cell to another depending on their size and localization, but Fig. 8a-c shows that in the abstract vector space composed by these characteristics (referred to as the stress space hereafter), points representing cells are distinctly clustering according to morpholog- 

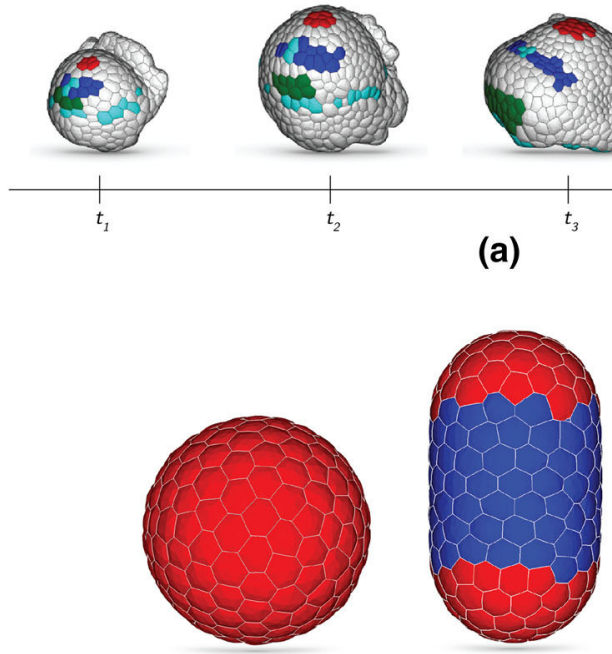

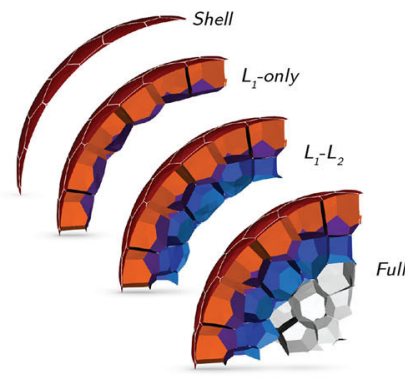

(c)

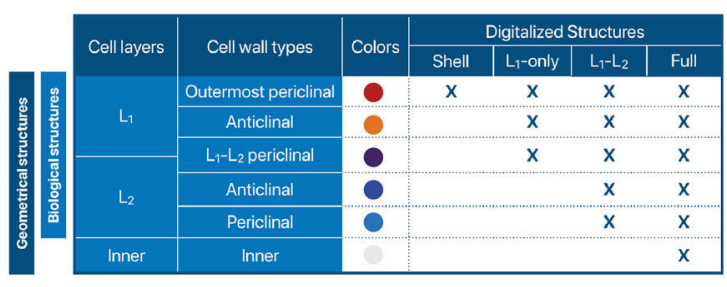

(d)

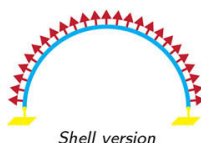

Shell version

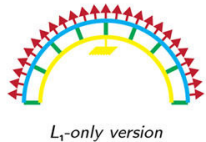

$L_{1}$-only version

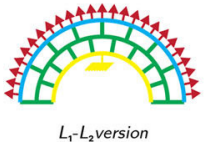

(e)

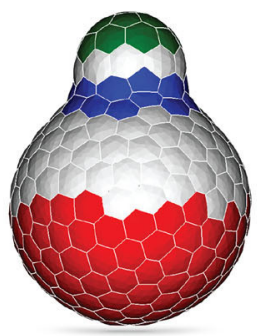

(b)

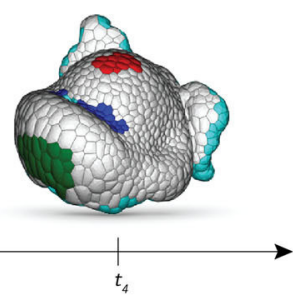

(a) 


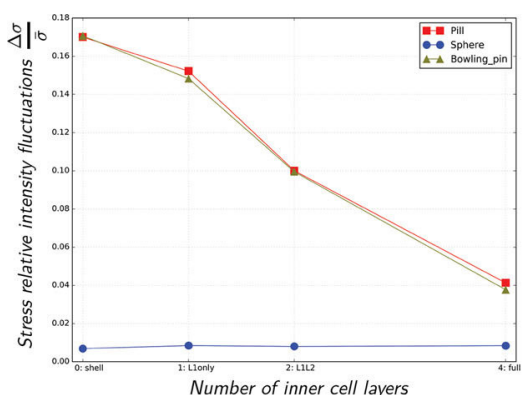

(a)

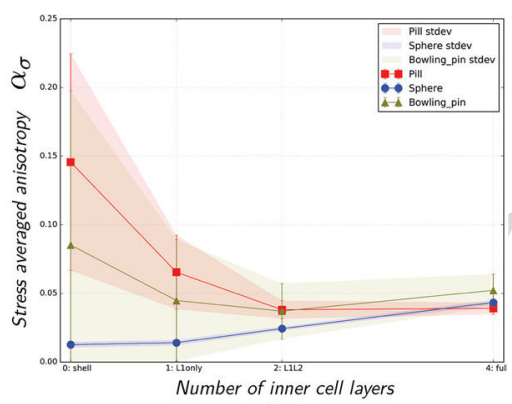

(b)

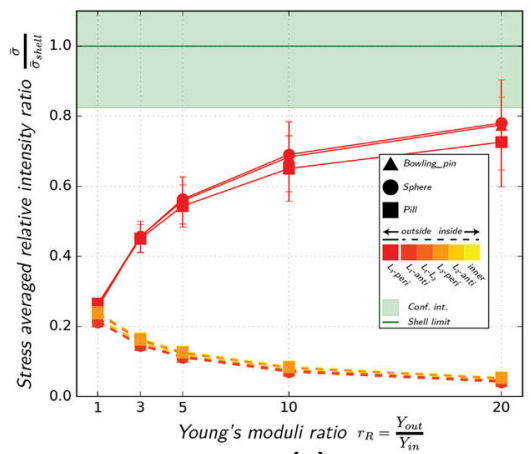

(c)

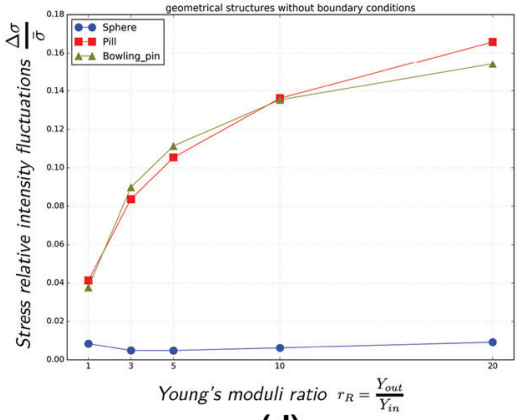

(d)

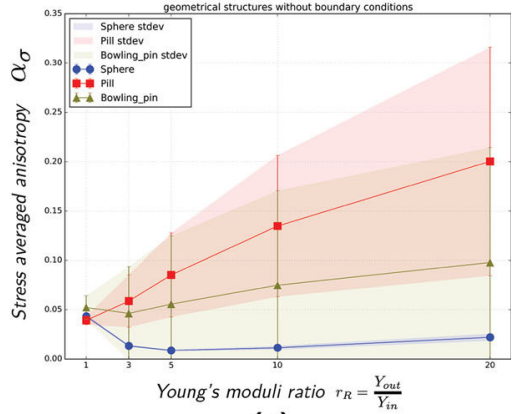

(e)

Fig. 3 Influence of the structure depth and cell wall stiffness heterogeneity on the outermost periclinal stress pattern on geometrical structures. a Cell-to-cell relative stress intensity fluctuations (standard deviation divided by mean value) as a function of the structures depth for each shape (sphere, pill, bowling pin). For each cell of the epidermis ( $L_{1}$ layer), the mean relative stress intensity is computed. b Averaged stress anisotropy as a function of the structures depth for each shape (sphere, pill, bowling pin). Error bars stand for standard deviation over the epidermis cells. $\mathbf{c}$ Ratio between the relative stress intensity in the full structure over the relative stress intensity in the shell structure as a function of rigidity differential between the outermost cell walls $\left(L_{1}-\right.$ peri $)$ and the inner ones in the full structure. Each curve corresponds to a specific cell wall type. Error bars stands for standard deviation. d Cell-to-cell relative stress intensity fluctuations in full structures (standard deviation divided by mean value) as a function of rigidity differential between the outermost cell walls $\left(L_{1}-\right.$ peri $)$ and the inner one. e Averaged stress anisotropy as a function of rigidity differential between the outermost cell walls $\left(L_{1}-\right.$ peri $\left.i\right)$ and the inner one. Error bars stand for standard deviations (Color figure online) 
ical identities. More precisely, Fig. 8a, b shows that on the fourth time step realistic structure, where morphological features-such as outgrowing initia and creases-are well established, point representing epidermal cells are clustering in distinct groups corresponding to these significant morphological features. Figure $8 \mathrm{c}$ shows that a clear distinction can also be made between epithelial cells and inner cells in the stress space.

This last sorting suggests that epithelial cells are experiencing higher stresses in their periclinal plane than along their apicobasal direction, whereas inner cells are experiencing the opposite configuration. This dichotomy originates from the rigidity differential between the inner and outer walls: stiffer outer walls bear more stress and therefore drag the corresponding point away from the first bisectrix toward the right. In simulations where the rigidity ratio $Y_{\text {out }} / Y_{\text {in }}$ was changed, the sorting was impacted accordingly (data not shown).

\section{Distinct morphological fates correlate with specific trajectories within the stress} space In order to further assess the correlation between plant tissues morphological features and clustering of cells in the stress space, we looked at the point clouds corresponding to older structures (the first, second and third structures of our realistic time series). Since we were able to reconstruct the lineage of epidermal cells across our time series, we could follow the trajectories of each cell line (i.e., a mother cell on the first time step and its clones in the following ones) within the stress space as the young flower is shaping itself. In Fig. 8d, e, we see that cell lines leading to the formation of specific zones follow coherent trajectories, specific to distinct zones; In Fig. 8e, cells forming the central zone of the meristem do not move much from the center of the plane, whereas cells forming the crease and the sepal abaxial side are, respectively, moving toward the bottom right corner and the top left corner of the frame. Similarly, in Fig. 8c, trajectories of cell lines forming the crease feature a drastic and coherent shift of trajectories between the third and fourth time step, concomitant with the crease formation.

\section{Discussion}

Robust and reproducible morphogenesis relies on control mechanisms where geometry feeds back onto growth. Such regulation loops are required to translate geometry into information perceptible by cells. In plants, turgor-induced stresses emerge as such geometry-related information fields.

In order to model and simulate properly plant morphogenesis, we need to understand how this transduction from tissue geometry to mechanical stress works. In particular, how parameters such as the accuracy of tissue descriptions (number and shapes of cells, depth of the considered tissues, rheological properties) as well as the assumptions underlying simulations (e.g., boundary conditions) influence computed stress fields and therefore may modulate geometrical information available to cells.

In order to have an informative outermost periclinal stress field, cell wall rigidity must be heterogeneous The informativeness of a stress pattern can be quantified by its spatial directional variabilities, Fig. 3. For a given asymmetric shape (e.g., pill, bowling pin...) the variability of an informative turgor-induced stress pattern should 


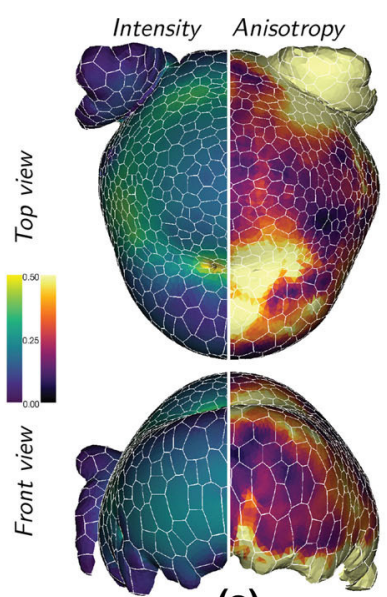

(a)

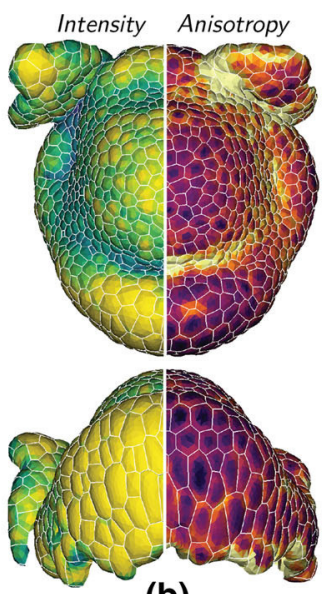

(b)

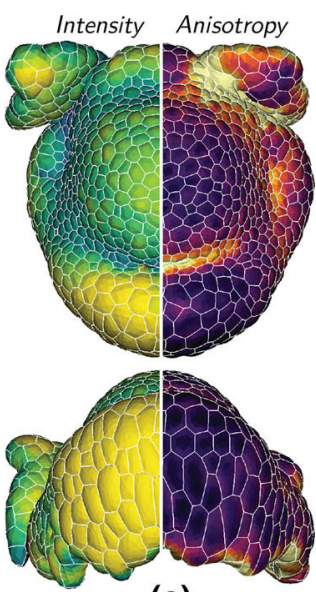

(c)

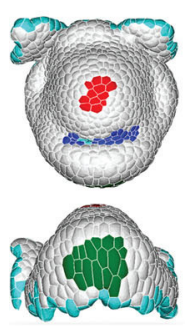

(d)

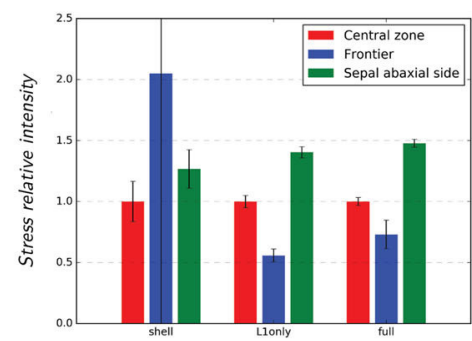

(e)

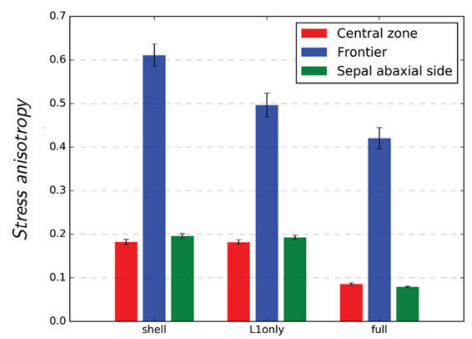

(f)

Fig. 4 Outermost periclinal stress pattern characteristics on a realistic structure (fourth step of the time series). a-c Relative stress intensity (left-hand side) and anisotropy (right-hand side) heat maps computed on the three different version of the same time point: (a shell, b $L_{1}-$ only $\mathbf{c} L_{1}-L_{2}$. The corresponding maps of main stress directions are given as supplementary Fig. 1. d Visualization of the three morphological zones of interest: Central Zone in red, Frontier in blue Sepal Abaxial Zone in green. e, f Outermost periclinal stress invariants - $\mathbf{e}$ intensity (normalized by the central zone value), $\mathbf{f}$ anisotropy - averaged over zones of interest for the three versions of the same structure. Left-hand side: Shell, middle: $L_{1}-$ only, right-hand side: $L_{1}-L_{2}$. Error bars stand for standard deviations

correlate with that of the surface curvature field. The higher the variabilities of the stress pattern, the more informative it is, i.e., discrimination between regions with different geometrical features is made easier.

In our simulations, two main parameters influenced the stress pattern variabilities: the number of considered inner cell layers, Fig. 3a, b, and the rigidity differential between the outermost cell walls and the inner ones, Fig. 3d, e. While increasing the number of considered inner cell layers decreased the outermost stress pattern variability, rigidity differential between outer and inner walls increased it.

This can be qualitatively explained: by adding inner load-bearing elements to a $2 \mathrm{D}$ pressurized shell one enables the stresses to distribute in 3D. This redistribution of stresses between outer and inner walls is done in proportion to the walls rigidity (i.e., stiffer ones bear proportionally more stress than softer ones, see Fig. 3c). 3D struc- 


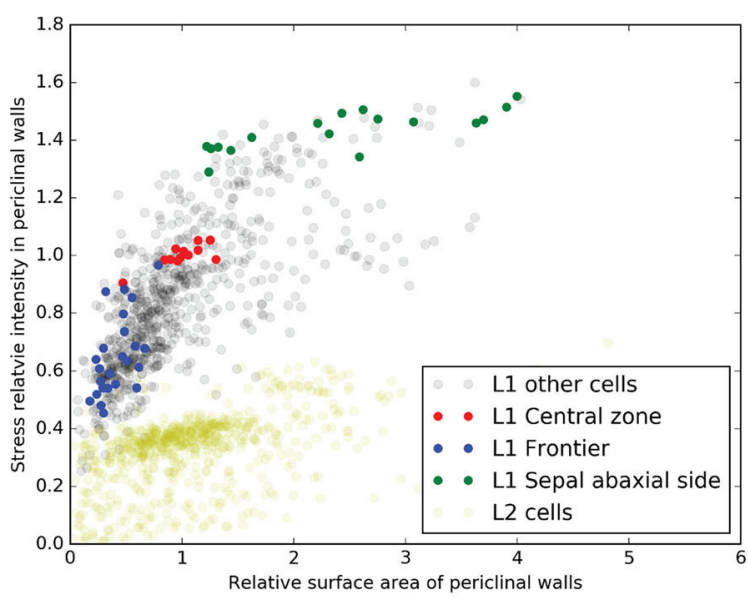

(a) (b)

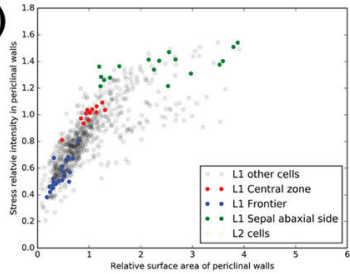

(c)

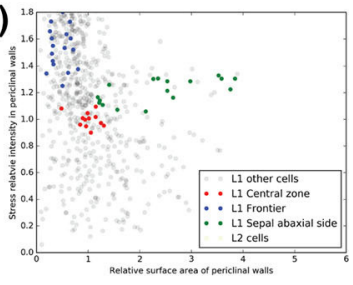

Fig. 5 Relationship between relative stress intensity in outermost periclinal walls and epidermal cells surface areas (fourth step of the realistic time series). Relative stress intensity (normalized by its mean value in the central zone) as a function of cells periclinal surface area (normalized by its mean value in the central zone) for the three versions of the structure: $\mathbf{a} L_{1}-L_{2} \mathbf{b} L_{1}$-only $\mathbf{c S h e l l}$. Each point corresponds to a single cell. The abscissa corresponds to the surface area of the cells outermost wall (outerpericlinal for $L_{1}$ cells $L_{1}-L_{2}-$ periclinal for $L_{2}$ ones, see table (c) ) Color code corresponds to various zones on interest in the $L_{1}$ defined on $\mathbf{c}$

tures with similar walls everywhere tend to behave mechanically as 3D homogeneous continua, in which stress fields are known to be shape-independent and thus noninformative. Rigidity differential between the outermost surface and the inner walls diminishes the mechanical influence of the latter and therefore enhances contrast (and informativeness) of the stress pattern.

For the sake of simplicity, we only considered isotropic cell walls here and the only envisioned heterogeneity was between outer and inner walls. In vivo, plant cell walls can be highly anisotropic and heterogeneous on the tissue surface (Milani et al. 2011). A follow-up question could address the influence of these more realistic and more complex modulations of the rigidity on the stress patterns.

Negatively curved regions, such as frontiers between organs, experience lowintensity and high-anisotropy stresses Considering the anticlinal walls in realistic structures helped us resolve an apparent paradox. According to the pressurized shell model and our simulations on shells (Fig. 4a), cells lying in the creases between the central zone and lateral organs experience high-intensity stresses compared to neighboring zones. Since plant cells growth is fueled by turgor-induced pressure, this would suggest fast growth of such cells, which is not observed experimentally (Burian et al. 2013; Kwiatkowska 2004).

When anticlinal walls are taken into consideration, stress intensity in the frontier zone decreases down from a relative maximum to a relative minimum, Fig. 4e. This resolves the previously mentioned paradox: when anticlinal walls are considered, creases between organs undergo low-intensity stresses compatible with small growth 


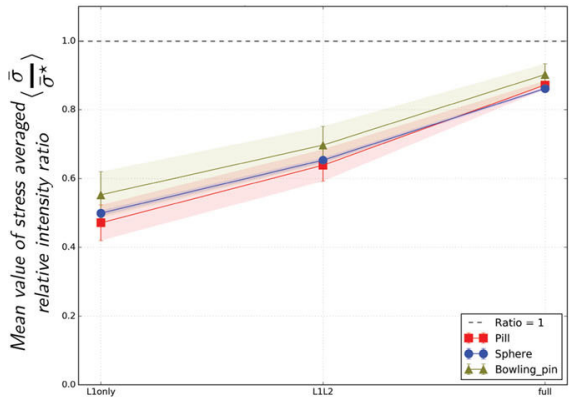

(a)

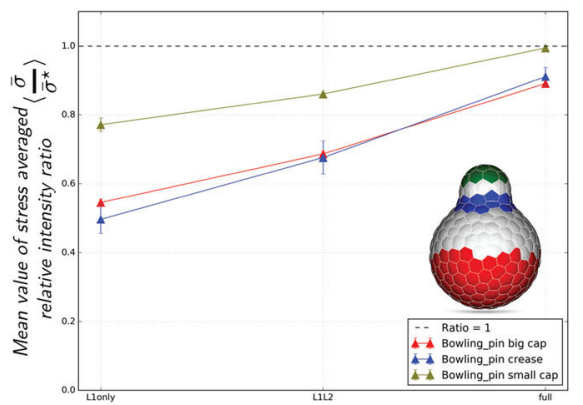

(c)

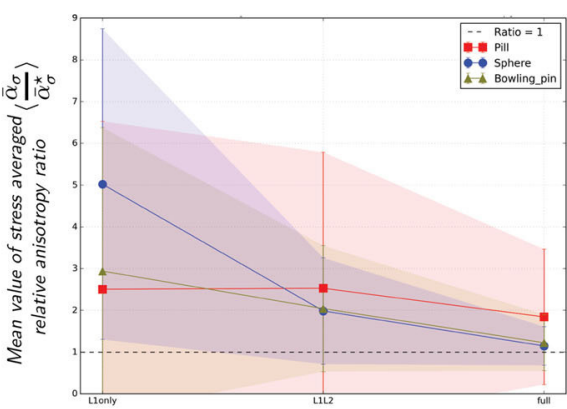

(b)

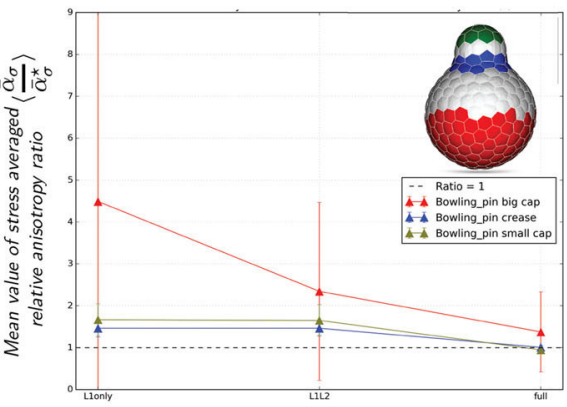

(d)

Fig. 6 Influence of the boundary conditions on the outermost periclinal stress pattern undergone by geometrical structures with inner walls. a Mean values of the outermost periclinal stress intensity ratio between structures with fixed innermost walls and a full one without fixed walls. b Mean values of the outermost periclinal stress anisotropy ratio between structures with fixed innermost walls and a full one without fixed walls. On both subfigures, each curve corresponds to a specific shape (red: Pill, blue: Sphere green: bowling pin) and each points corresponds to a specific version of the considered shape with an increasing number of considered inner layers from left to right. Error bars stands for standard deviation over all the considered epidermal cells. c, $\mathbf{d}$ Same figures as $\mathbf{a}, \mathbf{b}$ but only on the bowling pin structure. This time each graph refers to a specific zone on the structure

rates measured in such regions (Burian et al. 2013; Kwiatkowska 2004). Qualitatively, the inversion of colors between the left-hand side panels of Fig. 4a-c suggests that all zones with negative Gaussian curvature experience this low-intensity stresses when inner walls are considered. Note that this is coherent with classic wounding experiments performed on sunflowers (Dumais and Steele 2000): when sunflower meristems are cut, the resulting wounds are wider in the central zone than in the surrounding crease.

One possible explanation could be that anticlinal walls in these negatively curved regions act as the main load-bearing elements against turgor-induced forces as the apicobasal alignment of stresses in anticlinal walls suggests, Fig. 7a-d. However, Fig. 7e, f shows that anticlinal stresses borne by cells in the frontier zone are also lower than those borne by cells in other regions of interest. Anticlinal walls are therefore not the main load-bearing elements in the frontier zone, this is confirmed by Fig. 8c: cells of the frontier zone, as all epidermal cells, experience bigger stresses on their 


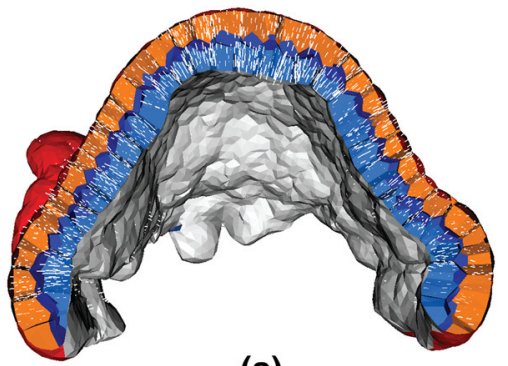

(a)

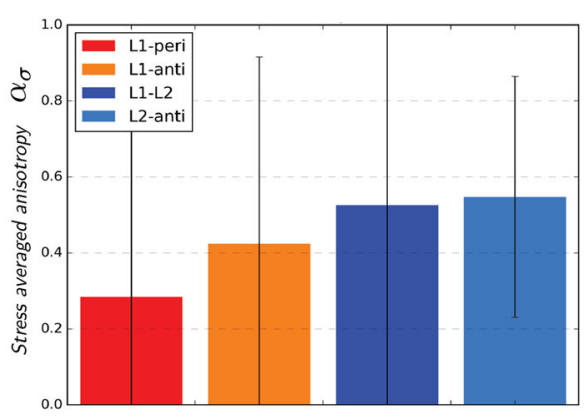

(c)

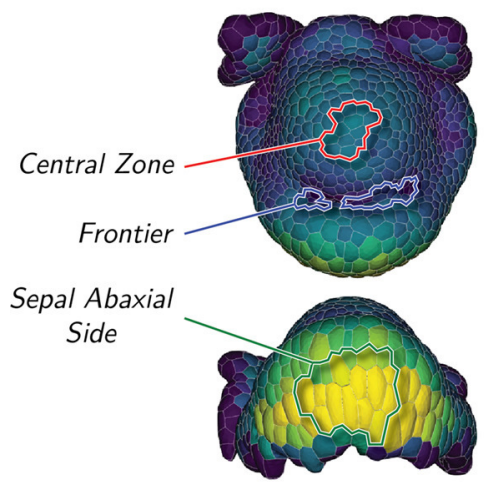

(e)

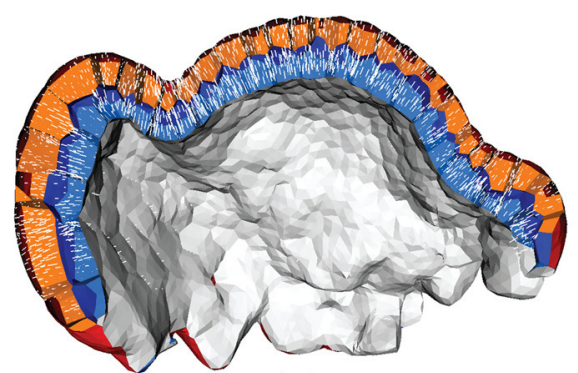

(b)

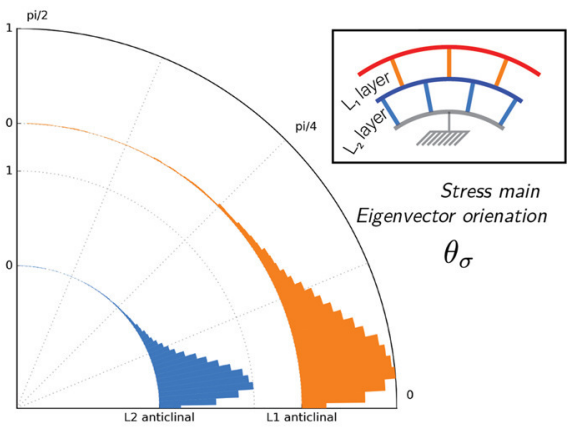

(d)

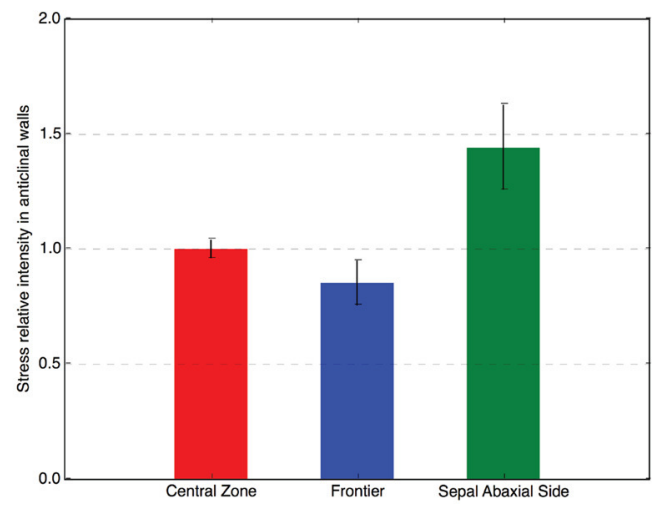

(f)

Fig. 7 Inner walls stress field characteristics on realistic structure (fourth step of the time series, $L_{1}-L_{2}$ version). a, b Anticlinal stress orientation (direction of the maximal stress eigenvalue) in the epidermis ( $L_{1}$, orange) and sub-epidermis layers $\left(L_{2}\right.$, blue). c Histograms of inner stress anisotropy per cell wall types. Error bars stands for standard deviations. d Angular distributions of the anticlinal stress eigenvectors associated with the maximal eigenvalue. $\theta_{\sigma}=0$ corresponds to the apicobasal direction and $\theta_{\sigma}=\pi / 2$ to the periclinal plane. e Heat map of the averaged relative anticlinal stress intensity per cells in the epidermis. f Histograms of the averaged relative anticlinal stress intensity per cells in the epidermis

outermost periclinal face than on their anticlinal ones. Note that periclinal surface area of cells in the frontier zone is rather small compared to the rest of the tissue. 


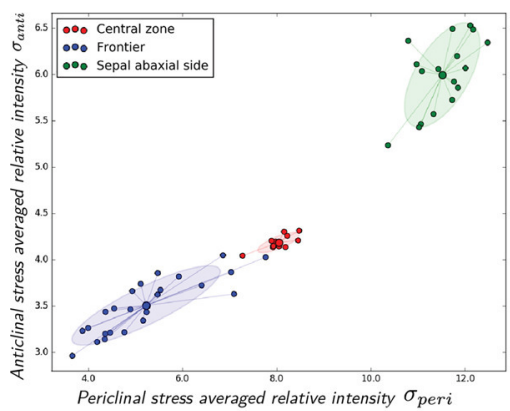

(a)

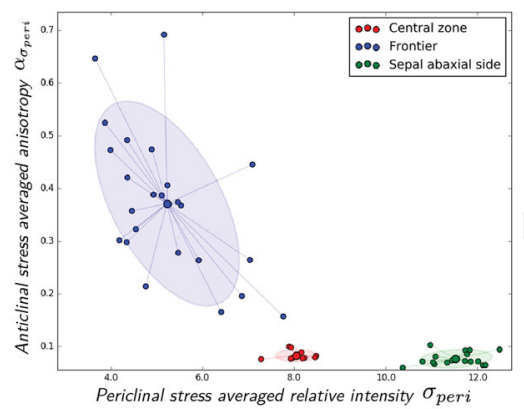

(b)

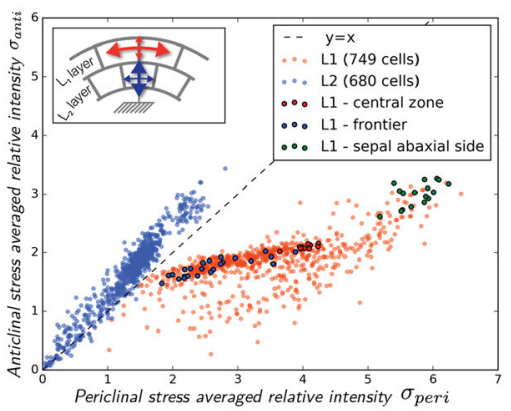

(c)

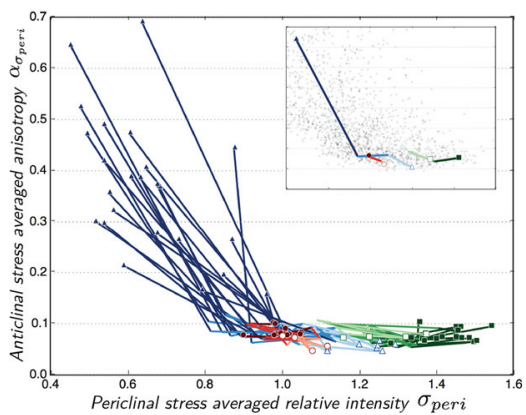

(d)

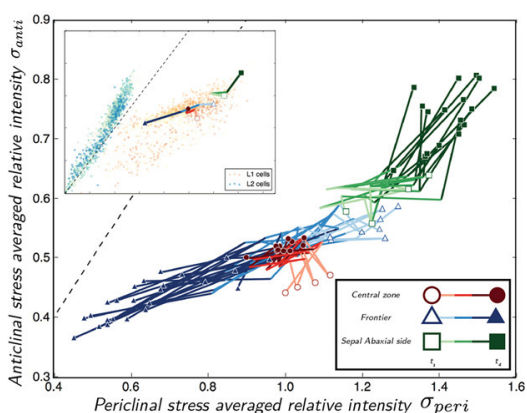

(e)

Fig. 8 Characterization of cells mechanical state on realistic structure (fourth step of the time series). a, b Point clouds representing the averaged stress state of each cell of the three zones of interest defined in Fig. 4d. In a, the coordinates of these points correspond to the mean stress intensity in the periclinal walls (abscissa) and anticlinal walls (ordinates). In $\mathbf{b}$, the coordinates of these points correspond to the mean stress intensity (abscissa) and anisotropy (ordinates) in the periclinal walls. The ellipses figure the covariant matrices of the various point clouds $\mathbf{d}$ 3D structures capturing the early sepal formation. Four time steps taken every $12 \mathrm{~h}$. On time step $t_{4}$ three topographic zones are defined on the epidermis $\left(L_{1}\right)$ layer: the central zone (CZ-red), the frontier ( $\mathrm{F}$-blue) and the abaxial side of the biggest sepal (AS-violet). Lineage of these zones is tracked back in older time steps and mother cells are displayed with the same color code as daughters. $\mathbf{f}$ Time evolution of cells forming the zones of interest in the peri-/anti- clinal relative stress intensities space. Each cell of each zone for of interest is depicted by a point in the plane. The lines between points represent their evolution from one time step to the next. For clarity reasons, only cells of the first $\left(t_{1}\right)$ and last $\left(t_{4}\right)$ time points are shown. Insert: global view of trajectories in the phase plane where $L_{1}$ (green-blue dots) and $L_{2}$ (orange) cells are superimposed 
It remains to be seen if cell division-and the resulting increase in anticlinal walls density-account for this decrease in periclinal stress intensity.

Turgor-induced stress reflects both cell-scale and tissue-scale geometries Previous numerical approaches performed on artificial structures (Mosca et al. 2017) and on realistic ones (reconstructed from confocal images of sepals Mosca et al. 2017 and seedlings Bassel et al. 2014) revealed a connection between cell geometrical features and mechanical stresses. Moreover, the authors of Mosca et al. (2017), Bassel et al. (2014) suggested that such a connection could play a central regulatory role during morphogenesis. In this study, the various versions of the same realistic structure suggest that this connection between cell geometry and mechanical stresses is rooted in the static geometrical and topological properties of tissues: in the 3D cases $\left(L_{1}\right.$-only $\left.L_{1}-L_{2}\right)$, we observed a correlation between outermost periclinal stress intensity and cell surface area, see Fig. 5a, b. Moreover, the specific shape of these point clouds suggests a saturating relation. This result gains interest when put in the context of a recent study on hypocotyls (Sapala et al. 2018). Focusing on epidermal cells, the authors obtained a similar saturation distribution profile between pavement cell periclinal surface areas and the intensity of the stress they experience, see figure 3-H of Sapala et al. (2018). Put together with our own results, this suggests that a general relationship between cells size and the intensity of the turgor-induced stress could be important for the regulation of growth in plants.

The fact that this correlation only appears when anticlinal walls considered (no correlation observed in Fig. 5c) reveals that the mechanical stresses experienced by cells encompass a short-range component, related to cell shape, mixed with a largescale component, related to tissue geometry and captured by the pressurized shell model.

Influence of boundary conditions From a global standpoint, Fig. 6a, b shows that fixing inner walls results, respectively, in an underestimation of stress intensity and an overestimation of stress anisotropy on the outermost surface. This seems intuitive: fixed inner points ultimately anchor the loaded structure and end up carrying some of the pressure-induced load, explaining a drop in the global elastic stress response. Figure $4 \mathrm{~b}, \mathrm{c}$ shows that within each epithelial cell, the increase in anisotropy is mostly located near their anticlinal walls. When the innermost points of the structure are fixed, anticlinal walls lose some of their freedom of movement. This constrains the in-plane deformations of cells as well as the resulting elastic stresses within the outermost periclinal walls of the epidermis. The closer to the surface the fixed points, the more obvious this effect.

Depending on their curvature, various zones on the same structure are not similarly impacted by boundary conditions. Comparison between the small and big caps of the bowling pin structures, see Fig. $6 \mathrm{c}$, d, suggests that as the ratio $h / R$-where $h$ and $R$, respectively, stand for the thickness of the structure (i.e., the distance between the outermost, free, surface and the innermost, fixed, one)—increases, the influence of fixed boundary conditions on the outermost stress pattern vanishes. This can be qualitatively explained: the radius of curvature is the characteristic length that most influences the outermost stress pattern (c.f. Laplace's law for spherical pressurized membrane: $P \propto R^{-1}$ —with $P$ the pressure differential between the two sides of the 
membrane). When the boundary is far enough from the outermost surface (i.e., farther than this characteristic length), their influence is negligible. In realistic structures with fixed thickness, we therefore foresee that boundary conditions will impact flat zones more than highly curved ones.

In the previous subsection, we suggested that fixed boundary conditions generated a short-range component-reflecting cell shape—within the turgor-induced stress field. Combined with the qualitative analysis presented above, this entails that the relative importance of this component-compared to the long range one-should increase as tissues grow from small bulging primordia into flat structures as leaves and sepals.

Naturally, the biological relevance of fixed boundary conditions is questionable. From a mechanical point of view, fixing walls below a given depth is similar to assuming infinitely stiffer tissues below this depth compared to the above ones. While this has not been described in fast growing tissues, the development of vasculature in more differentiated organs could provide such a rigidity gradient. Stiffening more or less deeply cell walls could be a way to modulate the relative importance of the cell-shape and the tissue-geometry-related components within the outermost stress field.

\section{Turgor-induced stress as a source of positional information field in plant tissues} In conclusion, our results show that within morphologically distinct regions, cells featured specific and coherent stress characteristics. This enables an efficient clustering of cells in a low-dimensional (3D) abstract vector space, Fig. 8a-c. The pronounced segregation between clusters in this stress space suggests that stress-sensitive cells could plausibly discriminate between them and adapt their fate accordingly. This gives credit to the mechanical spatial awareness hypothesis.

Extending this analysis to the whole time series composed by our realistic structures, we see that cell lines can be visualized as trajectories in the stress space. Figure 8d, e shows that epithelial cell lines developing toward specific morphological features correspond to coherent streams in the stress space. This analysis points at a number of interesting aspects. For instance, one can follow the trajectory corresponding to the formation of the frontier zone between the sepal and the meristem-blue lines in Fig. 8e. The corresponding points steadily move toward the first bisector over the course of the whole process. As mentioned earlier, the first bisector of the $\left\{\sigma_{\text {peri }}, \sigma_{\text {anti }}\right\}$-plane discriminates strongly between epithelial cells and inner ones, see Fig. 8c. This suggests that cells forming a crease are evolving toward a mechanical stress environment similar to the one of inner cells. In the future, this analysis could be refined by considering more stress characteristics. The corresponding clusters and trajectories would consequently enable the distinction between more subtle morphological features.

Acknowledgements The authors would like to thank: Guillaume Cerutti for his advice on mesh generation, Arezki Boudaoud and Olivier Hamant for their useful comments and also Jonathan Legrand and Pradeep Das for kindly providing the time series of segmented 3D images of the growing flower bud used in this analysis. The authors also thank Gabriella Mosca for informal discussions and comments on the work.

Funding Funding was provided by European Research Council (Grant No. Morphodynamics) and Inria Project Lab (Grant No. Morphogenetics). 


\section{References}

Ali O, Mirabet V, Godin C, Traas J (2014) Physical models of plant development. Annu Rev Cell Dev Biol 30:59-78

Armezzani A, Abad U, Ali O, Robin AA, Vachez L, Larrieu A, Mellerowicz EJ, Taconnat L, Battu V, Stanislas T, Liu M, Vernoux T, Traas J, Sassi M (2018) Transcriptional induction of cell wall remodelling genes is coupled to microtubule-driven growth isotropy at the shoot apex in Arabidopsis. Development 145(11):dev162255-11

Bassel GW, Stamm P, Mosca G, de Reuille PB, Gibbs DJ, Winter R, Janka A, Holdsworth MJ, Smith RS (2014) Mechanical constraints imposed by 3D cellular geometry and arrangement modulate growth patterns in the Arabidopsis embryo. Proc Natl Acad Sci USA 111(23):8685-8690

Beauzamy L, Louveaux M, Hamant O, Boudaoud A (2015) Mechanically, the shoot apical meristem of arabidopsis behaves like a shell inflated by a pressure of about $1 \mathrm{MPa}$. Front Plant Sci 6:1038

Boudon F, Chopard J, Ali O, Gilles B, Hamant O, Boudaoud A, Traas J, Godin C (2015) A computational framework for 3D mechanical modeling of plant morphogenesis with cellular resolution. PLoS Comput Biol 11(1):e1003950

Bozorg B, Krupinski P, Jönsson H (2014) Stress and strain provide positional and directional cues in development. PLoS Comput Biol 10(1):e1003410

Bozorg B, Krupinski P, Jönsson H (2016) A continuous growth model for plant tissue. Phys Biol 13(6):1-14 Burian A, Ludynia M, Uyttewaal M, Traas J, Boudaoud A, Hamant O, Kwiatkowska D (2013) A correlative microscopy approach relates microtubule behaviour, local organ geometry, and cell growth at the Arabidopsis shoot apical meristem. J Exp Bot 64(18):5753-5767

Cerutti G, Ali O, Godin C (2017) DRACO-STEM: an automatic tool to generate high-quality 3D meshes of shoot apical meristem tissue at cell resolution. Front Plant Sci 8:13-15

Chanliaud E, Burrows K, Jeronimidis G, Gidley M (2002) Mechanical properties of primary plant cell wall analogues. Planta 215(6):989-996

Chickarmane V, Roeder AHK, Tarr PT, Cunha A, Tobin C, Meyerowitz EM (2010) Computational morphodynamics: a modeling framework to understand plant growth. Annu Rev Plant Biol 61(1):65-87

Corson F, Hamant O, Bohn S, Traas J, Boudaoud A, Couder Y (2009) Turning a plant tissue into a living cell froth through isotropic growth. Proc Natil Acad Sci 106(21):8453

Dumais J (2007) Can mechanics control pattern formation in plants? Curr Opin Plant Biol 10(1):58-62

Dumais J, Steele CR (2000) New evidence for the role of mechanical forces in the shoot apical meristem. J Plant Growth Regul 19(1):7-18

Dumais J, Shaw SL, Steele CR, Long SR, Ray PM (2006) An anisotropic-viscoplastic model of plant cell morphogenesis by tip growth. Int J Dev Biol 50(2-3):209-222

Faure F, Duriez C, Delingette H, Allard J et al (2012) Sofa: a multi-model framework for interactive physical simulation. Springer, Berin

Fernandez R, Das P, Mirabet V, Moscardi E, Traas J, Verdeil J-L, Malandain G, Godin C (2010) imaging plant growth in 4d: robust tissue reconstruction and lineaging at cell resolution. Nat Methods 7(7):547-553

Geitmann A, Ortega JKE (2009) Mechanics and modeling of plant cell growth. Trends Plant Sci 14(9):467478

Green JBA, Sharpe J (2015) Positional information and reaction-diffusion: two big ideas in developmental biology combine. Development 142(7):1203-1211

Hamant O, Heisler MG, Jonsson H, Krupinski P, Uyttewaal M, Bokov P, Corson F, Sahlin P, Boudaoud A, Meyerowitz EM, Couder Y, Traas J (2008) Developmental patterning by mechanical signals in Arabidopsis. Science 322(5908):1650-1655

Hamant O, Meyerowitz EM, Traas J (2011) Is cell polarity under mechanical control in plants? Plant Signal Behav 6(1):137-139

Hejnowicz Z (2005) Autonomous changes in the orientation of cortical microtubules underlying the helicoidal cell wall of the sunflower hypocotyl epidermis: spatial variation translated into temporal changes. Protoplasma 225(3-4):243-256

Hejnowicz Z, Rusin A, Rusin T (2000) Tensile tissue stress affects the orientation of cortical microtubules in the epidermis of sunflower hypocotyl. J Plant Growth Regul 19(1):31-44

Hervieux N, Dumond M, Sapala A, Routier-Kierzkowska A-L, Kierzkowski D, Roeder AHK, Smith RS, Boudaoud A, Hamant O (2016) A mechanical feedback restricts sepal growth and shape in Arabidopsis. Curr Biol 26(8):1019-1028 
Irvine KD, Shraiman Boris I (2017) Mechanical control of growth: ideas, facts and challenges. Development 144:4238-4248

Keller R (2012) Physical biology returns to morphogenesis. Science 338(6104):201-203

Kwiatkowska D (2004) Surface growth at the reproductive shoot apex of Arabidopsis thaliana pin-formed 1 and wild type. Journal of experimental botany 55(399):1021-1032

Kierzkowski D, Nakayama N, Routier-Kierzkowska A-L, Weber A, Bayer E, Schorderet M, Reinhardt D, Kuhlemeier C, Smith RS (2012) Elastic domains regulate growth and organogenesis in the plant shoot apical meristem. Science 335(6):1096

Ladoux B, Mège RM (2017) Mechanobiology of collective cell behaviours. Nat Rev Mol Cell Biol 18(12):743-757

Landau LD, Lifshitz EM (1959) Course of theoretical physics vol 7: theory and elasticity

Lintilhac PM, Vesecky TB (1984) Stress-induced alignment of division plane in plant-tissues grown-invitro. Nature 307(5949):363-364

Louveaux M, Julien J-D, Mirabet V, Boudaoud A, Hamant O (2016) Cell division plane orientation based on tensile stress in Arabidopsis thaliana. Proc Natl Acad Sci USA 113(30):E4294-303

Milani P, Gholamirad M, Traas J, Arnéodo A, Boudaoud A, Argoul F, Hamant O (2011) Invivo analysis of local wall stiffness at the shoot apical meristem in Arabidopsis using atomic force microscopy. Plant J 67(6):1116-1123

Mosca G, Sapala A, Strauss S, Routier-Kierzkowska A-L, Smith RS (2017) On the micro-indentation of plant cells in a tissue context. Phys Biol 14(1):015003-015012

Oliveri H, Traas J, Godin C, Ali O (2018) Regulation of plant cell wall stiffness by mechanical stress: a mesoscale physical model. J Math Biol 78:625-653

Ortega JK (1985) Augmented growth equation for cell wall expansion. Plant Physiol 79(1):318-320

Pradal C, Dufour-Kowalski S, Boudon F, Fournier C, Godin C (2008) OpenAlea: a visual programming and component-based software platform for plant modelling. Funct Plant Biol 35(9-10):751-760

Sampathkumar A, Krupinski P, Wightman R, Milani P, Berquand A, Boudaoud A, Hamant O, Jönsson H, Meyerowitz EM (2014) Subcellular and supracellular mechanical stress prescribes cytoskeleton behavior in Arabidopsiscotyledon pavement cells. eLife 3:584-20

Sapala A, Runions A, Routier-Kierzkowska A-L, Das Gupta M, Hong L, Hofhuis H, Verger S, Mosca G, Li C-B, Hay A, Hamant O, Roeder AH, Tsiantis M, Prusinkiewicz P, Smith RS (2018) Why plants make puzzle cells, and how their shape emerges. eLife 7:2061

Shraiman BI (2005) Mechanical feedback as a possible regulator of tissue growth. Proc Natl Acad Sci 102(9):3318-3323

Théry M, Racine V, Piel M, Pépin A, Dimitrov A, Chen Y, Sibarita J-B, Bornens M (2006) Anisotropy of cell adhesive microenvironment governs cell internal organization and orientation of polarity. Proc Natl Acad Sci 103(52):19771-19776

Thompson D'Arcy W (1917) On growth and form. Cambridge University Press, Cambridge

Tournier M, Nesme M, Gilles B, Faure F (2015) Stable constrained dynamics. ACM Trans Graphics (TOG) 34(4): 132

Turing FRSAM (1952) The chemical basis of morphogenesis. Phil Trans R Soc Lond B 237(641):37-72

Uyttewaal M, Burian A, Alim K, Landrein B, Borowska-Wykręt D, Dedieu A, Peaucelle A, Ludynia M, Traas J, Boudaoud A, Kwiatkowska D, Hamant O (2012) Mechanical stress acts via katanin to amplify differences in growth rate between adjacent cells in Arabidopsis. Cell 149(2):439-451

Vining KH, Mooney DJ (2017) Mechanical forces direct stem cell behaviour in development and regeneration. Nat Rev Mol Cell Biol 18(12):728-742

Vogel G (2013) How do organs know when they have reached the right size? Science 340(6137):1156-1157

Weber A, Braybrook S, Huflejt M, Mosca G, Routier-Kierzkowska AL, Smith RS (2015) Measuring the mechanical properties of plant cells by combining micro-indentation with osmotic treatments. J Exp Bot 66:3229-3241

Wolpert L (1969) Positional information and the spatial pattern of cellular differentiation. J Theor Biol 25(1): $1-47$

Publisher's Note Springer Nature remains neutral with regard to jurisdictional claims in published maps and institutional affiliations. 